\title{
Risk of cervical cancer after completed post-treatment follow-up of cervical intraepithelial neoplasia: population based cohort study
}

\author{
(c) $(1)$ (8) OPEN ACCESS
}

\author{
Matejka Rebolj postdoctoral researcher ${ }^{12}$, Theo Helmerhorst professor of gynaecology ${ }^{3}$, Dik \\ Habbema professor of medical decision sciences ${ }^{1}$, Caspar Looman statistician ${ }^{1}$, Rob Boer \\ informatician/statistician ${ }^{1}$, Joost van Rosmalen postdoctoral researcher ${ }^{1}$, Marjolein van Ballegooijen \\ associate professor of medical technology assessment ${ }^{1}$
}

'Department of Public Health, Erasmus MC, Rotterdam, Netherlands; ${ }^{2}$ Department of Public Health, University of Copenhagen, Copenhagen, Denmark; ${ }^{3}$ Department of Obstetrics and Gynaecology, Erasmus MC, Rotterdam, Netherlands

\begin{abstract}
Objective To compare the risk of cervical cancer in women with histologically confirmed cervical intraepithelial neoplasia who returned to routine screening after having completed post-treatment follow-up with consecutive normal smear test results with women with a normal primary smear test result.

Design Population based cohort study using data from a nationwide pathology register.

Setting The Netherlands, 1994 to 2006.

Population 38956 women with histologically confirmed intraepithelial neoplasia grades 1 to 3 with completed follow-up after treatment.

Intervention Routine post-treatment follow-up of cervical intraepithelial neoplasia, recommending smear tests at six, 12, and 24 months.

Main outcome measure Incidence of cervical cancer in the period from completed follow-up with negative test results after cervical intraepithelial neoplasia to the next primary test. 10-year hazard ratios were compared with periods after normal results for the primary smear test, adjusted for year in follow-up.

Results 20 cervical cancers were diagnosed during 56956 woman years after completed follow-up of cervical intraepithelial neoplasia, whereas 1613 cervical cancers were diagnosed during 25020697 woman years after a normal primary smear test result. The incidence of 35.1 (95\% confidence interval 21.4 to 54.2) per 100000 woman years and 6.4 (6.1 to 6.8) per 100000 woman years, respectively, led to an adjusted hazard ratio of 4.2 (95\% confidence interval 2.7 to 6.5$)$ for periods after completed follow-up compared with periods after normal primary smear test results. This hazard ratio was increased for all ages.
\end{abstract}

No significant difference in risk of cervical cancer was observed by grade of cervical intraepithelial neoplasia.

Conclusions An excess risk of cervical cancer previously observed for women treated for cervical intraepithelial neoplasia was also observed in the subgroup of women who completed their post-treatment follow-up with three consecutive normal smear test results. The overall corrected risk of cervical cancer in these women was increased fourfold 35 cases per 100000 woman years) compared with women with normal primary smear test results (6 per 100000 woman years).

\section{Introduction}

The aim of cervical screening is to reduce the incidence of and mortality from cervical cancer. This is predominantly achieved by identifying and treating cervical intraepithelial neoplasia. Although treatment is highly effective, ${ }^{1}$ affected women remain at higher risk of cervical cancer than women in the general population. ${ }^{2-6}$ Consequently, follow-up after treatment to identify residual and recurrent lesions is usually recommended.

The intensity of follow-up testing after treatment for cervical intraepithelial neoplasia varies by country. For example, biennial testing for 25 years is recommended in Sweden, ${ }^{7}$ and yearly testing for 20 years is recommended in the United States, ${ }^{8}$ for 10 years in the United Kingdom, ${ }^{9}$ and for two years in Denmark, ${ }^{10}$ Slovenia, ${ }^{11}$ and the Netherlands. ${ }^{12}$ The piloted scheme in the United Kingdom now recommends follow-up with cytology and testing for human papillomavirus six months after treatment. ${ }^{13}$ In general, women with normal smear test results at follow-up are recommended to resume routine 
screening, with intervals varying between three and five years in most European countries. ${ }^{14}$ This is the same recommendation as for women with a normal primary smear test result, and would imply that after negative test results during post-treatment follow-up the risk is back to "normal." However, little is known about whether this is the case. ${ }^{5}{ }^{15}$ This can be tested only with detailed data, which are often not available.

We determined the risk of cervical cancer in women with histologically confirmed cervical intraepithelial neoplasia who completed post-treatment follow-up with negative test results according to one of the internationally least intensive follow-up recommendations - those of the Netherlands, compared with the risk in women with a normal primary smear test result. The analysis was based on highly complete national histopathology and cytopathology data from the Netherlands.

\section{Methods}

\section{Recommendations for follow-up in the Netherlands}

In the Netherlands, women with primary smear test results showing moderate or severe dyskaryosis, as well as women with smear test results showing persistent borderline or mild dyskaryosis are referred for colposcopy. Women with histologically confirmed cervical intraepithelial neoplasia grade 1 are recommended to have a colposcopy and a smear test in 12 months and are treated if particular conditions are met-for example, according to the size or position of the lesion, persistence, and when adequate follow-up cannot be guaranteed. ${ }^{12}{ }^{16}$ Although the guidelines argue for a conservative approach to treatment of small grade 2 lesions in young women, those with histologically confirmed grade 2 and grade 3 lesions are in general immediately treated. These women are recommended for cytological follow-up at six, 12, and 24 months. If any follow-up smear test result is at least borderline dyskaryotic, the women are recommended to undergo colposcopy. If the recommended three consecutive follow-up smear tests all provide normal results, the women are recommended to rejoin the regular screening programme, with repeat testing at five yearly intervals from age 30 to 60 years. ${ }^{12}$ After treatment of adenocarcinoma in situ, however, women remain in intensified follow-up for five years.

\section{Data source}

We retrieved data on all cervix uteri cytological and histological tests in the Netherlands registered until 31 March 2007 from the Dutch nationwide network and register of histopathology and cytopathology (PALGA). ${ }^{17}$ This registration began in the 1970s and has had virtually complete (about 99\%) coverage of pathology laboratories since $1991 .^{18} 19$ The pathology register is used primarily for revealing the total individual history of pathological investigations to pathologists when evaluating tissue or cells. It contains diagnoses that were communicated to treating doctors, and therefore reflects the clinical practice. During the period investigated, Dutch laboratories used predominantly conventional cytology.

In the pathology register, women are identified by their sex, birth date, and the first four letters of their maiden name. This identification string enables the linkage of different tests belonging to the same woman, and therefore also to follow individual testing histories (dates and diagnoses). We avoided the problem of false identity matches, which is present with such identification, ${ }^{20}$ by excluding women with $0.5 \%$ of the most common four letter combinations in maiden names, which corresponds to excluding about $30 \%$ of women. ${ }^{21}$ We made the reasonable assumption of no association between the measures we were interested in and having a common maiden name. A comparison with the laboratory data from Screening Region West of the Netherlands, in which the identification is complete, suggested that our method of excluding women with $0.5 \%$ of the most common surnames is adequate for calculating relative indicators. ${ }^{22}$

\section{Definition of cervical cancer}

We identified women with cervical cancer by selecting excerpts that included pathology codes for cervical cancer based on the Systematised Nomenclature of Medicine. ${ }^{18}$ These codes are sometimes also used for lesions that are not clearly invasive, or when there is doubt that the primary location was the cervix. Consequently, based on these codes the number of women with incident cervical cancer seems substantially higher than the number recorded in the Netherlands cancer registry. For women with cervical cancer codes in the pathology register between 1 January 1994 and 31 December 2006, we reviewed the conclusions (free text) of all registered histology reports. In case the codes differed from the pathologist's conclusion, we used the latter. A comparison with the data from the Netherlands cancer registry showed that after this review our edited pathology register data contained about $7 \%$ more cases of cervical cancer than the Netherlands cancer registry. The excess incidence in our data, however, was not clustered in a particular calendar year and it was observed in all age groups up to 75 years. ${ }^{22}$

\section{Definition of a primary test}

We defined a primary test as a non-secondary test. A secondary or follow-up test was defined as a histological or a cytological test taken within four years after a previous abnormal or inadequate test result, unless follow-up of the abnormal or inadequate test result had already been completed according to the guidelines. ${ }^{123} \mathrm{We}$ defined an abnormal test result as at least borderline dyskaryosis on cytology and its equivalent on histology. Completed follow-up would be, for example, two consecutive normal smear test results after a borderline dyskaryotic result, or three consecutive normal smear test results after histologically confirmed cervical intraepithelial neoplasia. We considered smear test results registered shortly after a previous smear test ( $\leq 4$ days) as duplicates, and these were excluded. By using these definitions we organised registered individual screening histories into testing episodes. An episode was defined as starting with a primary test (a smear or a biopsy) followed by secondary tests in case the primary smear test result was abnormal. Thus an episode lasted for one test if the primary test result was normal, otherwise until the follow-up was completed according to the guidelines, or if four years had passed since the last test.

\section{Definition of completed negative follow-up of histologically confirmed cervical intraepithelial neoplasia}

We identified all episodes with histologically confirmed cervical intraepithelial neoplasia grades 1,2, or 3 and no cervical cancer diagnosis, and thereafter classified the episodes according to the maximum diagnostic grade within the episode. Type of treatment is not registered consistently in the pathology register and could therefore not be used in the analysis. In the main analysis, we considered all episodes of cervical intraepithelial neoplasia that ended with three consecutive normal smear test results - that is, completed negative test results at follow-up 
(figure $\downarrow$ ). This was also the case for cervical intraepithelial neoplasia grade 1, although the guidelines recommended only one follow-up smear test. If new abnormal test results were encountered after the initial histological diagnosis of cervical intraepithelial neoplasia, we reset the counting of consecutive normal smear test results to zero. In a side analysis, we considered episodes of cervical intraepithelial neoplasia with completed negative test results at follow-up only if the timing of testing followed the recommendations. We allowed delays of up to six months at each step. In this side analysis, the third consecutive normal smear test result must have been registered between 18 months (an accepted minimum) and 42 months ( 24 months recommended for follow-up plus three delays of six months accepted at most) after the last abnormal smear test or biopsy result within the episode.

\section{Statistical analysis}

We observed the incidence of cervical cancer after completion of negative post-treatment follow-up compared with the incidence after any normal primary smear test. Woman years at risk were counted from the primary test of the current episode onwards. At present in the Netherlands invitations for screening are sent at fixed five year intervals when women turn 30, 35, $40,45,50,55$, and 60 years, and relatively few smears are taken outside of the screening programme. ${ }^{24}$ Counting the woman years from the primary test onwards therefore theoretically ensured that the next screening round after episodes of cervical intraepithelial neoplasia and after episodes with normal primary smear test results would coincide on average in about five years. The counting of woman years ended at the date of the first histologically proved diagnosis of cervical cancer if it was diagnosed in the next episode (cases), or otherwise at the primary test of the next episode, 31 December 2006, or on completion of a 10 year period, whichever came first. We censored the follow-up at 10 years because few woman years accrued beyond that period. The woman years at risk for episodes with completed negative test results at follow-up are represented by $a+b$ in the figure.

Because we manually checked all excerpts from the pathology register with the cervical cancer codes in the period between 1 January 1994 and 31 December 2006, the woman years at risk and cervical cancer cases were left censored until 1 January 1994. We additionally left censored woman years at risk in the episodes with cervical intraepithelial neoplasia ( $a$ in figure) from the primary test until the third consecutive normal smear test result because women were still being followed-up and no cervical cancer was by definition diagnosed during that period.

Based on these data, we calculated the yearly incidence of cervical cancer per 100000 woman years at risk and calculated 95\% Poisson confidence intervals using an exact method. ${ }^{25}$ After verifying that the yearly hazard ratios did not differ significantly, we calculated the 10 year overall hazard ratios by Cox regression with yearly follow-up intervals, and we corrected for year in follow-up. We did not additionally correct the hazard ratios for age at primary smear test because the numbers of diagnosed cervical cancers by age group (grouped as $\leq 29$ years, 30-49 years, and $\geq 50$ years) were small. The hazard ratios were calculated with $\mathrm{R}$ version 2.7.1.

\section{Results}

In total, 38956 episodes with histologically confirmed cervical intraepithelial neoplasia were completed with negative follow-up test results (table $1 \Downarrow$ ). For these episodes a primary smear test was taken at a mean age of 35.9 (SD 8.6) years. On average,
4.9 years of follow-up were available per episode during the first 10 years after the primary test. Of these, 3.4 years were left censored (accrued before the end of follow-up, or before 1994), leaving on average 1.5 woman years after the third consecutive normal smear test result available for the analysis. In total, 20 cases of cervical cancer were diagnosed in 56956 woman years in the analysis. The 7096816 primary smear tests with normal results were taken at a mean age of 41.9 (SD 10.9) years. On average, 4.1 years of follow-up accrued in the first 10 years for these smear tests, with 3.5 years on average available for analysis after left censoring of woman years before 1994. During the 25020697 woman years available for the analysis, 1613 cases of cervical cancer were diagnosed.

For women with completed negative follow-up test results after histologically proved cervical intraepithelial neoplasia, the yearly incidence of cervical cancer until the next episode were consistently increased compared with women with normal smear test results (table $2 \Downarrow$ ). The resulting 10 year hazard ratios were significantly increased in all three age groups, and the overall hazard ratio was 4.2 (95\% confidence interval 2.7 to 6.5 , table $3 \Downarrow)$. The results were not materially changed by including only the 23415 episodes of cervical intraepithelial neoplasia in which the timing of the normal test results followed the recommendations (38 040 woman years at risk and 13 cases of cervical cancer; footnote to table 1$)$. In this case, the 10 year hazard ratio was 4.0 (2.3 to 6.9).

For the comparison of the risk by grade of cervical intraepithelial neoplasia (table $4 \Downarrow$ ), among the 38956 episodes with completed negative follow-up test results, in 8837 episodes (23\%) the most severe diagnosis was cervical intraepithelial neoplasia grade 1 , in 9020 episodes (23\%) it was grade 2, and in 21099 episodes $(54 \%)$ it was grade 3 . The 10 year hazard ratio of cervical cancer after detection of grade 1 compared with grade 3 lesions was 1.3 (95\% confidence interval 0.4 to 3.7 ) and after detection of grade 2 compared with grade 3 lesions was 1.4 (0.5 to 4.2$)$.

\section{Discussion}

Compared with women with normal primary smear test results the overall risk of cervical cancer in women who had three consecutive normal smear test results during post-treatment follow-up for cervical intraepithelial neoplasia was increased fourfold (35 cases per 100000 woman years). This increased risk could be observed regardless of the woman's age and the grade of neoplasia. These results have implications for women treated for cervical intraepithelial neoplasia, although how the excess risk could be decreased is not straightforward.

\section{Limitations and strengths of the study}

Our analysis has some limitations. Firstly, owing to incomplete registration of the treatments we were not able to determine how many women with cervical intraepithelial neoplasia were treated. Nevertheless, Dutch guidelines recommend treatment of cervical intraepithelial neoplasia grade 3 and, with some exceptions, grade 2 lesions, and watchful waiting with treatment if necessary for grade 1 lesions. ${ }^{12}{ }^{16}$ Histological confirmation of cervical intraepithelial neoplasia, treatment, and follow-up smear tests are all undertaken by gynaecologists. The fact that women had completed post-histology follow-up with three smear tests may therefore suggest that the women received not only follow-up according to the recommendations but also treatment (including watchful waiting in the case of cervical intraepithelial neoplasia grade 1). In support of this assumption, we estimated from several Dutch national databases that nearly all women with grade 2 and grade 3 lesions must have been 
treated and that about $40 \%$ of women with grade 1 lesions must have been treated at some time. ${ }^{26}$ Secondly, although this was also one of the strengths of our analysis, we focused on determining the risk of cervical cancer in women with completed negative follow-up test results after a diagnosis of cervical intraepithelial neoplasia, whereas we did not determine the risk in women with incomplete follow-up. ${ }^{37}$ A correct assessment of their risk would require adjustments in study methods, and should be dealt with in a separate analysis.

Our analysis has other important strengths. Firstly, to our knowledge this is the first study to focus on women who completed the post-treatment follow-up according to a particular set of recommendations. We have learnt that the excess risk of cervical cancer after treatment of cervical intraepithelial neoplasia cannot be fully explained by lack of post-treatment follow-up. An earlier large population based study from British Columbia provided an estimate of the risk of cervical cancer in women who were under active surveillance - that is, they had at least one smear test carried out in a three year period, compared with all women after treatment of cervical intraepithelial neoplasia. ${ }^{5}$ Unlike in our study, a separate risk estimate was not presented for women who completed the recommended sequence of normal smear tests and could rejoin the routine screening programme. Secondly, we used a national population based pathology register with virtually complete coverage since 1991 and could therefore identify all women with cervical intraepithelial neoplasia and determine their follow-up histories. Thirdly, although our study was not the only one to do so, we used cervical cancer as the endpoint instead of cervical intraepithelial neoplasia or cervical cancer (typically with few cases of cancer). Our research group has shown that in evaluation of primary cervical screening using cervical intraepithelial neoplasia as the endpoint can give a substantially different estimate of the relative risk than using cervical cancer as the endpoint. ${ }^{28}$ This also seems to be the case after treatment. Without selecting on completed follow-up, other researchers have consistently shown that whereas the risk of residual or recurrent cervical intraepithelial neoplasia declines to the background level after the first few years after treatment, the risk of cervical cancer stays higher than in the background level. ${ }^{35}$ The same kind of contrast could be seen in the Netherlands. A recent study of 435 women with cervical intraepithelial neoplasia treated at three major hospitals or (a minority) randomised to condom use instead of being treated showed a virtually equal risk of cervical intraepithelial neoplasia grade 2 or worse after three consecutive normal post-treatment smear test results compared with women with normal cytology in population based screening. The logical conclusion of that study was to return women with completed negative follow-up test results to routine screening. ${ }^{19}$ Our study, however, found a continuing excess risk of cervical cancer until the next episode, suggesting an additional degree of complexity in decision making for women after treatment.

\section{Comparison with earlier studies}

The fourfold hazard ratio of cervical cancer in our study was higher than similar estimates in several other studies, in which the relative risk was typically less than three..$^{2-46}$ This difference can to some extent be explained by the types of comparison groups used. Many previous studies used the incidence in local general populations, including screened and unscreened women as the comparison. Had we also compared the incidence in treated women with that in the general population (about 12.5 per 100000 for women aged 25 to 64 years in 1994 to 2006, which represents the age range of $90 \%$ of the episodes included in our analysis along with about 10 years thereafter), ${ }^{29}{ }^{30}$ our estimate of the fourfold excess risk would be about halved. On the other hand, the study from British Columbia found a relative risk of about six, comparing with women at very low risk with at least three consecutive normal smear test results and no history of cervical intraepithelial neoplasia. ${ }^{5}$

Although the risk of cervical intraepithelial neoplasia progressing to cancer increases with increasing grade of neoplasia, ${ }^{31}$ in our data the risk of cervical cancer in women with treated grade 3 lesions did not seem to be higher than among women with treated grade 1 or 2 lesions. This corroborated the earlier findings from Finland. ${ }^{32}$ These results can be interpreted in several ways. Firstly, they could reflect a less aggressive clinical management of cervical intraepithelial neoplasia grades 1 and 2. However, some cervical intraepithelial neoplasia grade 1 and virtually all grade 2 lesions tend to be treated, ${ }^{26}{ }^{33}$ and even without treatment they are often regressive. ${ }^{31}{ }^{34}$ Secondly, the incidence of cervical cancer after cervical intraepithelial neoplasia observed in our study, of about 35 per 100000 woman years (table 2) is low compared with the observed progression rates of untreated cervical intraepithelial neoplasia grade 3 to cervical cancer, which is about $30 \%$ in 30 years. ${ }^{35}$ Therefore our data probably point to high effectiveness of the current clinical management of high grade cervical intraepithelial neoplasia in reducing the risk of cervical cancer.

\section{Causes of continued excess risk for cervical cancer}

Dutch recommendations requiring three consecutive normal smear test results before a treated woman can rejoin the routine screening programme are, internationally, some of the least intensive. Therefore it would be interesting to determine whether any additional normal smear test results beyond the third could help treated women in catching up with the low risk observed in women with normal primary smear test results. To study the risk of cervical cancer in the period after the subsequent normal primary smear test result, we used the same methodology as for the main analysis (see supplementary file). Among the 38956 women in our main analysis, only $8 \%$ had no additional normal smear test results; $23 \%$ had one, $18 \%$ had two, and $52 \%$ had at least three. In total, 39 cervical cancers were diagnosed in 232 428 woman years at risk, compared with 1226 cervical cancers in 20571145 woman years in the 5646137 episodes with normal primary smear test results without any previous abnormality. The resulting 10 year hazard ratio after additional normal smears was 3.6 (95\% confidence interval 2.6 to 5.0), which is only slightly lower than the hazard ratio of 4.2 after three consecutive normal smears test results (table 3 ). This is despite the fact that after having completed the recommended follow-up, women with previous cervical intraepithelial neoplasia continued to undergo cervical testing more often than untreated women (on average every 1.9 years versus every 4.3 years). If anything, the contrast in the risk of cervical cancer would be larger if the Netherlands used shorter screening intervals. The risk after routine screening with shorter intervals would be expected to be modestly lower, ${ }^{36}$ whereas the risk after treatment would be expected to be the same.

These puzzling additional data from women with intensive follow-up emphasise the need to investigate the causes of the excess risk of cervical cancer after treatment of cervical intraepithelial neoplasia. These causes are at present poorly understood and could not be evaluated directly in our study. The excess risk might be a consequence of missed residual neoplasia or of de novo incidence because treated women are a selected high risk group. Firstly, residual neoplasia might be 
missed because changes in tissue after treatment may decrease the sensitivity of cytology for cervical abnormalities. Two meta-analyses, however, evaluated cytology and human papillomavirus testing in primary screening and as follow-up after treatment of cervical intraepithelial neoplasia. ${ }^{37}$ The sensitivity of cytology for high grade cervical intraepithelial neoplasia seemed comparable in the two situations. Furthermore, detection of cervical cancer at an earlier stage in screened women than in the general population is one of the indicators of effective cervical screening. In the British Columbia study, for example, $77 \%$ of cervical cancers diagnosed after treatment of cervical intraepithelial neoplasia were stage 1 , compared with $53 \%$ diagnosed at stage 1 in the general population in the United States between 1998 and $2003 .{ }^{5}$ These findings suggest that after treatment of cervical intraepithelial neoplasia, cytology is similarly sensitive for early cancer as in primary screening. Nevertheless, the findings cannot rule out a possibility that some of the cervical cancers in women with several consecutive normal smear test results in our study developed from systematically missed residual lesions. If this is correct, then the use of more sensitive follow-up tests might have reduced the incidence of cervical cancer. Secondly, cervical cancers diagnosed after completed follow-up may have developed from de novo lesions because women treated for cervical intraepithelial neoplasia might have a higher background risk than the general population. Characteristics of women or their partners (for example, more frequent reinfections with human papillomavirus) or problems with the woman's immune system (for example, a relatively low capability to clear the infection compared with the general population) would lead to more frequent newly developed progressive cervical intraepithelial neoplasia. In this case, treated women may have benefitted from the more frequent screening observed in our data, and might further benefit from continued frequent screening.

A multicohort study observed the long term incidence of cervical intraepithelial neoplasia grade 2 or worse in Dutch women who were followed-up by frequent cytology and human papillomavirus testing for two years after treatment and then rejoined routine screening. Five to 21 years after treatment, $13 \%$ of these women had a human papillomavirus infection, ${ }^{19}$ compared with about $4-5 \%$ in the general Dutch population. ${ }^{38}$ The higher prevalence of infections seems compatible with both the residual lesions and the de novo incidence hypotheses. The study furthermore suggested that lesions may be picked up better by human papillomavirus testing than by cytology, in that fewer human papillomavirus tests would be needed to obtain the same protection as with cytology. ${ }^{19}$ This may mean that turning to human papillomavirus testing may be more efficient and possibly more effective than intensifying cytological follow-up. Because of relatively frequent positive human papillomavirus test results without underlying dysplasia, however, management of women with positive test results should be studied carefully.

\section{Follow-up recommendations after treatment}

Frequent follow-up with cytology is justifiable in the first two to three years after treatment, when most residual cervical intraepithelial neoplasia tends to be picked up. ${ }^{5}{ }^{19}$ After having observed that women remain at increased risk of cervical cancer for a long time after treatment, several authors have proposed that such women should be kept in long term frequent follow-up, ranging from 10 to 25 years,,$^{3-5}$ although the current trend in some European countries has been more towards shorter rather than longer follow-up. Our study alone, however, cannot give a straightforward answer to what should be recommended in the longer term. Several factors should be taken into account when deciding on the optimal length of post-treatment follow-up. In our study, treated women continued to be screened at short intervals, which may have helped reduce their burden of cervical cancer to an unknown degree. Although the remaining absolute risk observed from the third consecutive negative smear test result after treatment until the next episode (35 per 100000 woman years) was four times higher than in women without previous detection of cervical intraepithelial neoplasia, it was still low. Finally, the absolute risk of cervical cancer is expected to decrease in the coming decades as human papillomavirus based screening is routinely introduced, and substantial proportions of young birth cohorts are currently being vaccinated against two major carcinogenic human papillomavirus genotypes. As a consequence, we expect a reduction in the risk of cervical cancer after routine screening, and the number of women with treated cervical intraepithelial neoplasia may also decrease.

To determine the most optimal management, modelling is often used. Modelling may be helpful in exploring the consequences of the different hypotheses on the determinants of the observed excess risk. However, as we do not have conclusive data on why treated women are at increased risk despite relatively frequent screening, an effective and still efficient follow-up strategy cannot be derived from modelling. Only a randomised controlled trial embedded in a screening programme could give a more conclusive answer, although such a trial would probably necessitate inclusion of a prohibitive number of women.

In conclusion, an excess risk of cervical cancer that has been previously observed for the complete group of women treated for cervical intraepithelial neoplasia could also be observed in the subgroup of women who completed their post-treatment follow-up with three consecutive normal smear test results.

We thank Menno Dekker and Roel Faber for help with data management. Contributors: MR, MvB, and DH designed the study. MR, CL, and JvR analysed the data. All authors interpreted the results, drafted the manuscript, and decided to submit the manuscript. MR and MvB are the guarantors. All authors had full access to all of the data in the study and wrote the manuscript independent of the funder.

Funding: This study was funded by the Dutch National Institute for Public Health and the Environment (RIVM, grant No 3022/07 DG MS/CvB/NvN). The RIVM had no role in the design of the study; the collection, analysis, and interpretation of the data; the writing of the report; and the decision to submit the manuscript for publication. The researchers worked independently of the funders.

Competing interests: All authors have completed the ICMJE uniform disclosure form at www.icmje.org/coi_disclosure.pdf (available on request from the corresponding author) and declare that: MR is currently involved in a comparative study of new generation human papillomavirus tests, for which Roche Diagnostics, Genomica, Qiagen, and Gen-Probe provided assays and instrumentations. MR did not receive any salary, compensation, or bonuses for work on any of the projects from any of the companies, and does not hold companies' stock. Concerning the present paper, there has been no involvement or support from any of the companies. Since 1989 RB has been participating in the screening research group at the Department of Public Health of Erasmus MC. Between 2009 and 2012 he was also director of health economics at Cerner LifeSciences, which is a consultancy that mainly works for the pharmaceutical industry and is part of Cerner that mainly develops and markets healthcare information technology. This research and article were not funded or supported by Cerner or their clients. RB's institution has received a grant from Health Insurance Executive Board. MvB was the principal investigator until 2008 on a project on the cost effectiveness of human papillomavirus vaccination, financed by GSK (a pharmaceutical company that produces human papillomavirus vaccines against cervical 


\section{What is already known on this topic}

Treatment of screen detected cervical intraepithelial neoplasia is highly effective in preventing development of cervical cancer Treated women nevertheless remain at higher risk of cervical cancer than untreated women, and this could be due to lack of post-treatment follow-up

\section{What this study adds}

Regardless of women's age and grade of cervical intraepithelial neoplasia, the risk of cervical cancer after completed post-treatment follow-up (35 per 100000 woman years) remained about four times higher than in women with normal primary smear test results The excess risk after treatment of cervical intraepithelial neoplasia cannot be fully explained by lack of post-treatment follow-up

cancer). There has been no collaboration with or support from GSK for the present paper. The authors have no other relationships or activities that could appear to have influenced the submitted work.

Ethical approval: Data retrieval was approved by the board of PALGA, the Dutch nationwide network and register of histopathology and cytopathology.

Data sharing: No additional data available.

1 Soutter WP, de Barros LA, Fletcher A, Monaghan JM, Duncan ID, Paraskevaidis E, et al Invasive cervical cancer after conservative therapy for cervical intraepithelial neoplasia. Lancet 1997;349:978-80.

2 Kalliala I, Anttila A, Pukkala E, Nieminen P. Risk of cervical and other cancers after treatment of cervical intraepithelial neoplasia: retrospective cohort study. $B M J$ 2005:331:1183-5.

3 Soutter WP, Sasieni P, Panoskaltsis T. Long-term risk of invasive cervical cancer after treatment of squamous cervical intraepithelial neoplasia. Int J Cancer 2006;118:2048-55

4 Strander B, Andersson-Ellstrom A, Milsom I, Sparen P. Long term risk of invasive cancer after treatment for cervical intraepithelial neoplasia grade 3: population based cohort study. BMJ 2007:335:1077.

5 Melnikow J, McGahan C, Sawaya GF, Ehlen T, Coldman A. Cervical intraepithelial neoplasia outcomes after treatment: long-term follow-up from the British Columbia Cohort Study. J Natl Cancer Inst 2009;101:721-8.

6 Pettersson F, Malker B. Invasive carcinoma of the uterine cervix following diagnosis and treatment of in situ carcinoma. Record linkage study within a National Cancer Registry. Radiother Oncol 1989:16:115-20.

7 Swedish Society of Obstetricians and Gynecologists (SFOG). Guidelines for the management of cervical intraepithelial neoplasia 2010, English summary. 2011. www. sfog.se/ARG+intressegrupper/natupplaga/English_summeryARG63.pdf.

8 ACOG Practice Bulletin No 109. Cervical cytology screening. Obstet Gynecol 2009:114:1409-20

9 Luesley D, Leeson S, eds. Colposcopy and programme management: guidelines for the NHS cervical screening programme, 2edn. NHSCSP Publication No 20. NHS Cancer Screening Programmes, 2010. 2011. www.cancerscreening.nhs.uk/cervical/publications/ nhscsp20.pdf.

10 Kjeld Petersen L, Frøding L, Lidang M, Arenholt L. Post-conisation follow-up (in Danish). Dansk Selskab for Gynækologi og Obstetrik, December 2007. 201. www.dsog.dk/hindsgavl/ post konus kontrol guideline udkast.pdf.

11 Uršič-Vrščaj M, ed. Guidelines for comprehensive management of women with abnorma cervix uteri (in Slovenian). Ljubljana: Onkološki Inštitut, 2011. http://zora.onko-i.si/data/ 2011_Smernice_web.pdf.

12 Helmerhorst TJ, Wijnen JA. Guidelines for cervical cancer screening programme (in Dutch). Ned Tijdschr Obstet Gynaecol 1998;111:264-5.

13 NHS Cervical Screening Programme. HPV triage and test of cure protocol. 2011. www. cancerscreening.nhs.uk/cervical/hpv-triage-test-of-cure.html.

14 Anttila A, von Karsa L, Aasmaa A, Fender M, Patnick J, Rebolj M, et al. Cervical cancer screening policies and coverage in Europe. Eur J Cancer 2009;45:2649-58.

15 Ronco G, Sideri MG, Ciatto S. Cervical intraepithelial neoplasia and higher long term risk of cancer. BMJ 2007;335:1053-4.

16 Werkgroep Oncologische Gynaecologie. Cervical intraepithelial neoplasia (CIN), nationwide guidelines, version 1.1 (in Dutch). 2011. www.oncoline.nl/cervicale-intra-epithelialeneoplasie.

17 Prismant. Dutch Network and National Database for Pathology (PALGA): results of retrieval of cervix uteri tests until 31 March 2007. Prismant, 2007.

18 PALGA. Description of the PALGA system and computer network (in Dutch). 2011. www. palga.nl.

19 Kocken M, Helmerhorst TJ, Berkhof J, Louwers JA, Nobbenhuis MA, Bais AG, et al. Risk of recurrent high-grade cervical intraepithelial neoplasia after successful treatment: a long-term multi-cohort study. Lancet Oncol 2011;12:441-50.
20 Van den Brandt PA, Schouten LJ, Goldbohm RA, Dorant E, Hunen PM. Development of a record linkage protocol for use in the Dutch Cancer Registry for Epidemiological Research. Int J Epidemiol 1990;19:553-8.

21 Van den Akker-van Marle ME, van Ballegooijen M, Habbema JD. Low risk of cervical cancer during a long period after negative screening in the Netherlands. Br J Cancer 2003;88:1054-7.

22 Rebolj M. Recent developments in the Dutch cervical cancer screening programme (PhD Thesis). Rotterdam: Department of Public Health, Erasmus MC, 2008. http://repub.eur. $\mathrm{nl} /$ resource/publication:14190/index.html.

23 Hanselaar AGJM, ed. CISOE-A in pictures (CD Rom, in Dutch). University Medical center Nijmegen and Dutch Association of Pathologists, 1996.

24 Rebolj M, van Ballegooijen M, Berkers LM, Habbema D. Monitoring a national cancer prevention program: successful changes in cervical cancer screening in the Netherlands. Int J Cancer 2007;120:806-12.

25 Ulm K. A simple method to calculate the confidence interval of a standardized mortality ratio (SMR). Am J Epidemiol 1990;131:373-5.

26 Rebolj M, Bais AG, van Ballegooijen M, Boer R, Meerding WJ, Helmerhorst TJ, et al. Human papillomavirus triage of women with persistent borderline or mildly dyskaryotic smears: comparison of costs and side effects of three alternative strategies. Int J Cancer 2007:121:1529-35.

27 Cecchini S, Ciatto S, lossa A, Ventura L, Zappa M. Re: Cervical intraepithelial neoplasia outcomes after treatment: long-term follow-up from the British Columbia Cohort Study. $J$ Natl Cancer Inst 2009;101:1429-30.

28 Rebolj M, van Ballegooijen M, Lynge E, Looman C, Essink-Bot ML, Boer R, et al. Incidence of cervical cancer after several negative smear results by age 50 : prospective observational study. BMJ 2009;338:b1354.

29 Dutch Comprehensive Cancer Centres. Cancer Registry Online: incidence of cervical cancer. 2011. www.ikcnet.nl/page.php?id=2902\&nav_id=114.

30 Dutch Central Bureau of Statistics. Population: sex, age, marital status and region per 1 January. 2011. http://statline.cbs.nl.

31 Ostor AG. Natural history of cervical intraepithelial neoplasia: a critical review. Int J Gynecol Pathol 1993;12:186-92.

32 Kalliala I, Nieminen P, Dyba T, Pukkala E, Anttila A. Cancer free survival after CIN treatment: comparisons of treatment methods and histology. Gynecol Oncol 2007;105:228-33.

33 Leinonen M, Nieminen P, Kotaniemi-Talonen L, Malila N, Tarkkanen J, Laurila P, et al. Age-specific evaluation of primary human papillomavirus screening vs conventional cytology in a randomized setting. J Natl Cancer Inst 2009;101:1612-23.

34 Castle PE, Schiffman M, Wheeler CM, Solomon D. Evidence for frequent regression of cervical intraepithelial neoplasia-grade 2. Obstet Gynecol 2009;113:18-25.

35 McCredie MR, Sharples KJ, Paul C, Baranyai J, Medley G, Jones RW, et al. Natural history of cervical neoplasia and risk of invasive cancer in women with cervical intraepithelial neoplasia 3: a retrospective cohort study. Lancet Oncol 2008:9:425-34.

36 Hakama M, Miller AB, Day NE, eds. Screening for cancer of the uterine cervix. International Agency for Research on Cancer, 1986.

37 Cuzick J, Arbyn M, Sankaranarayanan R, Tsu V, Ronco G, Mayrand MH, et al. Overview of human papillomavirus-based and other novel options for cervical cancer screening in developed and developing countries. Vaccine 2008;26(Suppl 10):K29-41.

38 Bulkmans NW, Berkhof J, Rozendaal L, van Kemenade FJ, Boeke AJ, Bulk S, et al. Human papillomavirus DNA testing for the detection of cervical intraepithelial neoplasia grade 3 and cancer: 5-year follow-up of a randomised controlled implementation trial. Lancet 2007;370:1764-72.

Accepted: 27 September 2012

\section{Cite this as: BMJ 2012:345:e6855}

This is an open-access article distributed under the terms of the Creative Commons Attribution Non-commercial License, which permits use, distribution, and reproduction in any medium, provided the original work is properly cited, the use is non commercial and is otherwise in compliance with the license. See: $\mathrm{http}: / /$ creativecommons.org/licenses/bync/2.0/ and http://creativecommons.org/licenses/by-nc/2.0/legalcode. 


\section{Tables}

Table 1 | Size of study groups

\begin{tabular}{lccc} 
Type of episode & $\begin{array}{c}\text { Mean (SD) age at primary smear } \\
\text { test (years) }\end{array}$ & No of episodes & No of woman years at risk \\
$\begin{array}{l}\text { CIN with completed negative } \\
\text { follow-up test results } \dagger\end{array}$ & $35.9(8.6)$ & 38956 & 56956 \\
\hline Normal primary smear test results & $41.9(10.9)$ & 7096816 & $20 \ddagger$ \\
\hline
\end{tabular}

$\mathrm{CIN}=$ histologically confirmed cervical intraepithelial neoplasia.

*Only non-censored woman years at risk are included.

tWhen episodes of CIN with completed negative follow-up results were considered only if the timing of follow-up tests followed recommendations (side analysis), 13 cases of cervical cancers were diagnosed in 38040 woman years within 23415 episodes that started at an mean age of 36.0 (SD 8.6 ) years.

fOne woman was treated for adenocarcinoma in situ. 
Table 2/ Yearly incidence rates of cervical cancer after completed negative follow-up results of histologically confirmed cervical intraepithelial neoplasia (CIN) and after normal primary smear test results

\begin{tabular}{|c|c|c|c|c|c|c|}
\hline \multirow{2}{*}{$\begin{array}{l}\text { Interval (years) } \\
\text { after primary } \\
\text { smear test }\end{array}$} & \multicolumn{3}{|c|}{ CIN with completed negative follow-up test results } & \multicolumn{3}{|c|}{ Normal primary smear test results } \\
\hline & Woman years & No of cases & $\begin{array}{l}\text { Incidence per } 100000 \\
\text { woman years }(95 \% \mathrm{Cl})\end{array}$ & Woman years & No of cases & $\begin{array}{l}\text { Incidence per } 100000 \\
\text { woman years }(95 \% \mathrm{Cl})\end{array}$ \\
\hline 0 to 1 & 76 & 0 & $0(0$ to 4853.8$)$ & 5442539 & 252 & $4.6(4.1$ to 5.2$)$ \\
\hline 1 to 2 & 3376 & 0 & $0(0$ to 109.3$)$ & 4829887 & 194 & $4.0(3.5$ to 4.6$)$ \\
\hline 2 to 3 & 9541 & 2 & $21.0(2.5$ to 75.7$)$ & 4158762 & 194 & 4.7 (4.0 to 5.4$)$ \\
\hline 3 to 4 & 11358 & 3 & 26.4 (5.4 to 77.2$)$ & 3277698 & 213 & 6.5 (5.7 to 7.4$)$ \\
\hline 4 to 5 & 10115 & 3 & 29.7 (6.1 to 86.7$)$ & 2451794 & 226 & 9.2 (8.1 to 10.5$)$ \\
\hline 5 to 6 & 7135 & 5 & $70.1(22.8$ to 163.5$)$ & 1433680 & 221 & $15.4(13.4$ to 17.6$)$ \\
\hline 6 to 7 & 5399 & 4 & $74.1(20.2$ to 189.7$)$ & 1081698 & 111 & $10.3(8.4$ to 12.4$)$ \\
\hline 7 to 8 & 4372 & 1 & $22.9(0.6$ to 127.4$)$ & 916117 & 85 & $9.3(7.4$ to 11.5$)$ \\
\hline 8 to 9 & 3272 & 2 & 61.1 (7.4 to 220.8$)$ & 776855 & 57 & 7.3 (5.6 to 9.5$)$ \\
\hline 9 to 10 & 2311 & 0 & $0(0$ to 159.6$)$ & 651666 & 60 & $9.2(7.0$ to 11.9$)$ \\
\hline Total $^{*}$ & 56956 & 20 & $35.1(21.4$ to 54.2$)$ & 25020697 & 1613 & $6.4(6.1$ to 6.8$)$ \\
\hline
\end{tabular}

*Total hazard ratio comparing incidence rates of cervical cancer 35.1 per 100000 woman years at risk and 6.4 per 100000 woman years at risk, corrected for year in follow-up, was 4.2 (95\% confidence interval 2.7 to 6.5 ; table 3$)$. 
Table 3 | Ten year hazard ratios of developing cervical cancer after negative follow-up test results following histologically confirmed cervical intraepithelial neoplasia, by age group

\begin{tabular}{lccc} 
Age (years) at primary smear test & Woman years at risk & No of cases & Hazard ratio* (95\% Cl) \\
$\leq 29$ & 12579 & 6 & $5.2(2.3$ to 11.7$)$ \\
\hline 30 to 49 & 39165 & 12 & $3.2(1.8$ to 5.6$)$ \\
\hline$\geq 50$ & 5212 & 2 & $6.8(1.7$ to 27.3$)$ \\
\hline Total & 56956 & 20 & $4.2(2.7$ to 6.5$)$ \\
\hline
\end{tabular}

${ }^{*}$ Compared with normal primary smear test results (see tables 1 and 2). Corrected for year in follow-up. 
Table 4| Ten year hazard ratios of developing cervical cancer after negative follow-up test results following histologically confirmed cervical intraepithelial neoplasia (CIN), for grades 1 and 2 lesions compared with grade 3

Grade of CIN Woman years at risk No of cases Hazard ratio* $(95 \% \mathrm{Cl})$

\begin{tabular}{lccc}
1 & 14482 & 6 & $1.3(0.4$ to 3.7$)$ \\
\hline 2 & 13752 & 6 & $1.4(0.5$ to 4.2$)$ \\
\hline 3 & 28722 & 8 & 1 (reference) \\
\hline Total & 56956 & 20 & - \\
\hline
\end{tabular}

${ }^{*}$ Corrected for year in follow-up. 


\section{Figure}

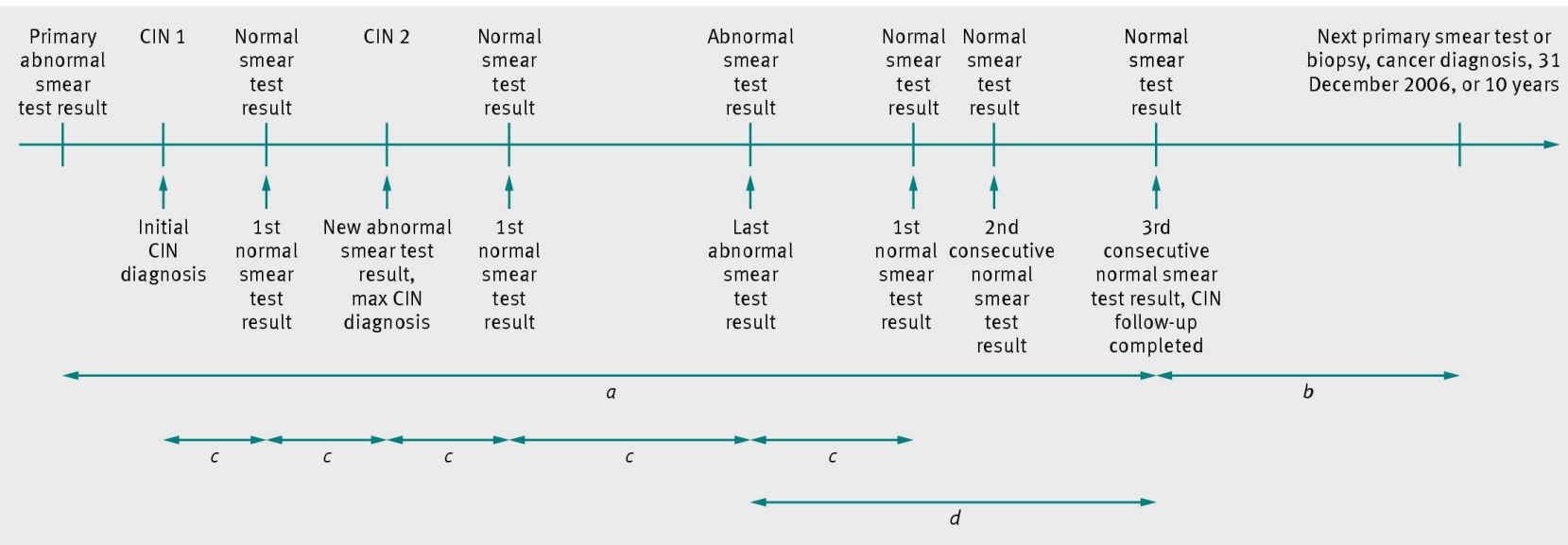

Definition of completed negative test results at follow-up after histologically confirmed cervical intraepithelial neoplasia and counting of woman years at risk for a hypothetical episode. $\mathrm{CIN}=$ histologically confirmed cervical intraepithelial neoplasia. $a=$ left censored (excluded) woman years at risk because of ongoing follow-up during which by definition no cervical cancer was diagnosed; $b$ =included woman years at risk. Because for all episodes woman years at risk were also left censored before 1 January 1994, episodes of cervical intraepithelial neoplasia with completed negative follow-up test results were included in the analysis if at least part of $b$ accrued after that date. Restrictions for side analysis (episodes of cervical intraepithelial neoplasia in which follow-up testing followed recommended timing): $c=$ maximum 12 months (recommended six months and an allowed delay of six months); $d=18$ to 42 months 